\title{
Safety, Efficacy, Pharmacokinetic and Pharmacodynamic evaluation of YF-H-2015005 for mobilizing Hematopoietic stem cells in Non-Hodgkin's Lymphoma Patients
}

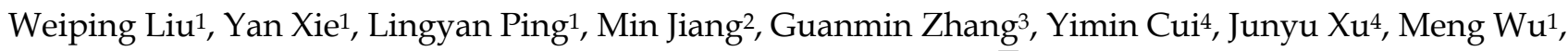
Xin Leng ${ }^{1}$, Xiaopei Wang ${ }^{1}$, Shufang Wang ${ }^{5}$, Jun Zhu ${ }^{1}$, Yuqin Song ${ }^{1 凶}$

1. Key Laboratory of Carcinogenesis and Translational Research (Ministry of Education), Department of Lymphoma, Peking University Cancer Hospital \& Institute, No.52 Fucheng Road, Haidian District, Beijing 100142, China.

2. Key Laboratory of Carcinogenesis and Translational Research (Ministry of Education), National Drug Clinical Trial Center (GCP Center), Peking University Cancer Hospital \& Institute, No.52 Fucheng Road, Haidian District, Beijing 100142, China.

3. Key Laboratory of Carcinogenesis and Translational Research (Ministry of Education), Department of Pharmacy, Peking University Cancer Hospital \& Institute, No.52 Fucheng Road, Haidian District, Beijing 100142, China

4. Peking University First Hospital, Department of Pharmacy, No.8 Xishiku Street, Xicheng District, Beijing 100034, China.

5. Hefei Yifan Biopharmaceuticals Inc., Intersection between Jinxiu avenue and qinglongtan road, Hefei 610000, China.

$\triangle$ Corresponding author: Yuqin Song, TEL: 8610 88196109; E-mail: songyuqin@pkuih.edu.cn; ORCID: 0000-0001-5451-6481.

(c) The author(s). This is an open access article distributed under the terms of the Creative Commons Attribution License (https://creativecommons.org/licenses/by/4.0/). See http://ivyspring.com/terms for full terms and conditions.

Received: 2020.05.27; Accepted: 2020.07.19; Published: 2020.07.25

\begin{abstract}
Background: Targeting the interaction between SDFI and CXCR4 may provide an opportunity to intervene in the hematopoietic stem cell mobilization process.

Aim: The present study aimed to investigate the safety, efficacy, pharmacokinetic and pharmacodynamic profiles of YF-H-2015005, a CXCR4 antagonist, for the mobilization of hematopoietic stem cells (HSCs). Methods: A total of 15 patients with non-Hodgkin's lymphoma (NHL) eligible for autologous hematopoietic stem cell transplantation were enrolled. All patients achieved a partial or complete remission after the first- or second-line therapy. Granulocyte colony stimulating factor (G-CSF) was given in the morning for 8 consecutive days, and $0.24 \mathrm{mg} / \mathrm{kg}$ YF-H-2015005 was subcutaneously administered in the evening of the $4^{\text {th }}$ day of G-CSF treatment for up to four days. Apheresis was performed 9-10 hours following each dose of YF-H-2015005. Results: YF-H-2015005 was rapidly absorbed and eliminated, with $\mathrm{T}_{\max }$ and $\mathrm{t}_{1 / 2}$ of 0.5 and $5.04 \pm 1.00$ hours, respectively. Moreover, the mean peripheral blood CD34+ cell counts were elevated by 2.0- to 2.9-fold from 2 to 24 hours, and reached the maximum level of $76.5 \pm$ 53.9 cells $/ \mathrm{kg}$ at 10 hours after YF-H-2015005 treatment. Fourteen (93\%) out of $15 \mathrm{NHL}$ patients achieved a minimum target of $\geq 2 \times 10^{6} / \mathrm{kg} \mathrm{CD} 34^{+}$cells. Furthermore, there was no grades 3-4 treatment-related adverse event observed among these patients. Conclusion: YF-H-2015005 can serve as a safe, effective agent in combination with G-CSF for CD34+ hematopoietic progenitor cell mobilization in NHL patients.
\end{abstract}

Key words: Hematopoietic Stem Cell Mobilization; Drug Evaluation; Pharmacokinetics; Safety; Lymphoma, Non-Hodgkin's

\section{Introduction}

The interaction of CXC chemokine receptor 4 (CXCR4) and its ligand CXCL12 (also referred as stromal cell-derived factor-1, SDF-1) plays an essential role in regulating the mobilization of hematopoietic stem cells (HSCs) [1,2]. SDF-1 is constitutively secreted in bone marrow stromal cells, while the glycoprotein-coupled receptor CXCR4 with SDF-1 homogeneity is mainly generated by HSCs [3,4]. Therefore, targeting the interaction between SDF1 and CXCR4 may provide an opportunity to intervene the 
mobilization of HSCs. Plerixafor, a CXCR4 antagonist, is able to enhance the steady-state release of SDF-1 into the bloodstream of both mice and non-human primates [5], and can lead to a 3- to 6-fold elevation in the absolute numbers of peripheral blood (PB) $\mathrm{CD} 34^{+}$ cells in healthy volunteers [6]. It is worth noting that plerixafor can exhibit high effectiveness on the mobilization of HSCs in patients with non-Hodgkin's lymphoma (NHL) or multiple myeloma [7]. In 2008, plerixafor was approved by the U.S. Food and Drug Administration for the mobilization of HSCs to the PB for collection and subsequent autologous hematopoietic stem cell transplantation (HSCT) [8].

YF-H-2015005 (1,1'-[1,4-phenylenebis(methylene)]-bis-1,4,8,11-tetraazacyclotetradecane) was a CXCR4 antagonist developed by Hefei Yifan Biopharmaceuticals Inc. (Anhui, China). It displayed the structural formula $\mathrm{C} 28 \mathrm{H} 54 \mathrm{~N} 8$, with a molecular mass of $502.79 \mathrm{~g} / \mathrm{mol}$. The molecular structure of YF-H2015005 was illustrated in Figure 1. Preclinical trials have demonstrated that YF-H-2015005 is effective and safe for enhancing the mobilization of HSCs. In this study, we determined the efficacy, safety, pharmacokinetic (PK) and pharmacodynamics (PD) profiles of YF-H-2015005 in combination with granulocyte colony stimulating factor (G-CSF) for $\mathrm{CD}^{+} 4^{+} \mathrm{HSC}$ mobilization in NHL patients. Furthermore, the enhancement effect of YF-H-2015005 on HSC mobilization in NHL patients was also evaluated.

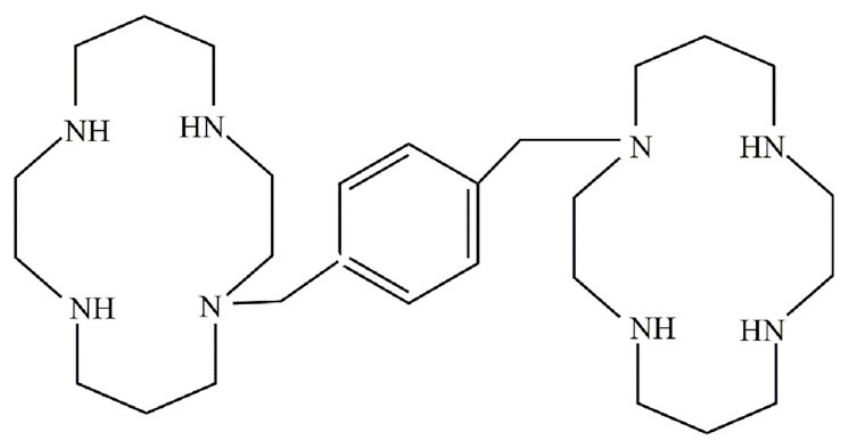

Figure 1. Structural formula of YF-H-2015005.

\section{Materials and Methods}

\section{Study design}

The ethical approval for this open-label, single arm study was obtained from the Ethics Committee of the Peking University Cancer Hospital and Institute (Beijing, China), and all procedures were conducted in accordance with the Declaration of Helsinki. All participants provided written informed consent prior to study enrollment. This study was registered on www.chinadrugtrials.org.cn (registration number: CTR20170925).
The inclusion criteria of this study were: (i) NHL patients eligible for autologous HSCT, males and females, aged 18-65 years; (ii) achieving a partial or complete remission after the first- or second-line therapy; (iii) an Eastern Cooperative Oncology Group (ECOG) performance status score of 0 or 1 ; (iv) did not overlap with the toxicity of other chemotherapeutic or anti-cancer drugs; (v) an actual body weight less than $175 \%$ of ideal body weight; (vi) negative for bone marrow involvement within 45 days prior to enrollment; and (vii) negative for human immunodeficiency and hepatitis viruses.

The main exclusion criteria were as follows: (i) chronic lymphocytic leukemia; (ii) failed previous HSC collections; (iii) history of allogeneic or autologous HSCT; (iv) history of pelvic radiotherapy; and (v) secondary central nervous system involvement. In addition, NHL patients with creatinine clearance rate $<50 \mathrm{~mL} / \mathrm{min}$ as well as abnormal liver function test results ( $>2.5$ times upper limit of normal) were excluded.

\section{Combination treatment of G-CSF and YF-H-2015005}

All patients were given a daily subcutaneous dose of G-CSF $(10 \mu \mathrm{g} / \mathrm{kg} /$ day $)$ each morning for 8 consecutive days. YF-H-2015005 with a dose of 0.24 $\mathrm{mg} / \mathrm{kg}$ was subcutaneously initiated on the evening of the $4^{\text {th }}$ day of G-CSF treatment and continued for up to 4 days. Apheresis was performed 9-10 hours following evening dose of YF-H-2015005. This process was continued daily for up to 4 days or until $\geq 2 \times 10^{6} / \mathrm{kg} \mathrm{CD} 34^{+}$cells were collected.

\section{PK and PD evaluation}

For PK analysis, blood samples were collected immediately before starting YF-H-2015005 treatment, and consecutively at $0.25,0.5,1,2,4,6,8,10,16$ and 24 hours following its administration and prior to apheresis. HSC apheresis was carried out using a COM.TEC instrument (Fresenius Kabi, Germany). Then, $4 \mathrm{~mL}$ of peripheral venous blood sample was collected into the heparin sodium tube at each time point of PK sampling. The tube was inverted 5 times and immediately placed on ice. After centrifuging at $3000 \mathrm{rpm}$ for 10 minutes, the resulting plasma was kept at $-70^{\circ} \mathrm{C}$. The plasma from each sample was split into two approximately equal aliquots.

All samples were evaluated by liquid chromatography coupled with tandem mass spectrometry (LC-MS/MS). The linear range of the assay was 2 to $1000 \mathrm{ng} / \mathrm{mL}$. The precision values of the quality control samples $(6,40,400$ and $750 \mathrm{ng} / \mathrm{mL})$ were all $\leq 8.5 \%$, with a bias of $-2.7 \sim 13.4 \%$. The PK parameters analyzed included the area under the 
curve from time 0 to $t\left(\mathrm{AUC}_{0-\mathrm{t}}\right)$, area under the curve from time 0 to infinity $\left(\mathrm{AUC}_{0-\infty}\right)$, maximum observed concentration $\left(\mathrm{C}_{\max }\right)$, time to achieve $\mathrm{C}_{\max }\left(\mathrm{T}_{\max }\right)$, apparent plasma clearance (CL), apparent volume of distribution $\left(\mathrm{V}_{\mathrm{z}}\right)$, and apparent terminal elimination half-life $\left(t_{1 / 2}\right)$. When multiple doses of YF-H-2015005 were administered, $\mathrm{PK}$ parameters were consisted of the steady-state trough concentration $\left(\mathrm{C}_{\text {ssmin }}\right)$, steady-state peak concentration $\left(\mathrm{C}_{\text {ssmax }}\right)$, average steady-state plasma drug concentration (Cav), steady-state area under the curve $\left(\mathrm{AUC}_{\mathrm{ss0}-24}\right)$, coefficient of fluctuation (Fl), and accumulation ratio (Ra). The values for all PK parameters were calculated with non-compartmental analysis using WinNonlin Professional version 6.3 software (Pharsight Corp, Mountain View, CA, USA).

For PD analysis, blood samples were collected immediately prior to dosing with YF-H-2015005 and then at 2, 4, 6, 8, 10, 16, and 24 hours after its administration. Four milliliter sample of peripheral venous blood was collected into the heparin sodium tube at each time point. The absolute number and percentage of CD34 cells were assessed by fluorescence-activated cell sorting method using a Beckman-Coulter FC500 flow cytometer and appropriate reagents.

\section{Safety and efficacy assessments}

The safety profiles were evaluated by clinical and laboratory parameters, including adverse event $(\mathrm{AE})$, serious $\mathrm{AE}$ (SAE), treatment-related $\mathrm{AE}$, treatment-related $\mathrm{SAE}$, and the AEs were monitored throughout the entire study period. All the clinical signs and symptoms were recorded, and a further laboratory examination or electrocardiogram was carried out (as dictated by the occurrence of $\mathrm{AE}$ ).

The efficacy endpoint was the proportion of NHL patients treated with YF-H-2015005 who achieved a minimum collection target of $\geq 2 \times 10^{6} / \mathrm{kg}$ $\mathrm{CD}^{+} 4^{+}$cells (actual body weight) within 4 days of apheresis.

\section{Statistical analysis}

All statistical analyses were conducted using IBM SPSS Statistics for Windows, Version 21.0 (IBM Corp., Armonk, New York, USA). Continuous variables were determined using the Student's t-test. Categorical variables were analyzed using the Pearson's chi-squared test or Fisher's exact test. All tests applied were two-tailed, and a $p$-value of less than 0.05 was deemed to be statistically significant.

\section{Results}

\section{Baseline characteristics}

In this study, 15 NHL patients (11 males and 4 females) were recruited, and none of them received fludrarabine or lenalidomide before HSC mobilization. The median age was 51 years. About $67 \%$ of patients had mature B-cell lymphoma. The median cycles of prior chemotherapy was 6 . None received radiotherapy. Table 1 revealed the demographic and disease characteristics of the enrolled NHL patients.

Table 1. Baseline characteristics of 15 patients treated with YF-H-2015005

\begin{tabular}{ll}
\hline Characteristics & All patients (\%) \\
\hline Age, years & \\
Mean (SD) & $47.8(10.8)$ \\
Median (range) & $51.0(32.0-64.0)$ \\
Ethnicity, n (\%) & $14(93.3)$ \\
Han & $1(6.7)$ \\
Others & \\
Gender, n (\%) & $11(73.3)$ \\
Male & $4(26.7)$ \\
Female & \\
Body weight, kg & $71.3(13.0)$ \\
Mean (SD) & $74.0(50.0-86.0)$ \\
Median (range) & \\
Pathology type & $1(6.7)$ \\
Lymphoblastic lymphoma & $4(26.7)$ \\
Peripheral T/NK cell lymphoma & $1 / 1 / 2$ \\
ALK-negative ALCL/ PTCL/ AITL & $10(66.6)$ \\
Mature B-cell lymphoma & $5 / 2 / 1 / 1 / 1$ \\
DLBCL/MCL/BL/tFL/ Unclassified & \\
Stage & $2(13.3)$ \\
I & $5(33.4)$ \\
II & $2(13.3)$ \\
III & $6(40.0)$ \\
IV & \\
Lines of chemotherapy & $10(66.7)$ \\
First line & $5(33.3)$ \\
Second line & \\
Cycles of chemotherapy & $7(3)$ \\
Mean (SD) & $6(4-12)$ \\
Median (range) & \\
Radiotherapy & \\
Yes & $15(0.0)$ \\
No & \\
Disease status \\
Complete remission
\end{tabular}

\section{PK and PD profiles of YF-H-2015005}

Table 2 summarized the PK data of YF-H2015005. The results demonstrated that YF-H-2015005 was rapidly absorbed in all 15 patients without a lag time after its first administration. Moreover, its median values of Tmax and median biological half-life $\left(t_{1 / 2}\right)$ were 0.5 and 5.04 hours, respectively. Figure 2 showed the mean plasma concentrations of YF-H-2015005 over time on both linear and semilogarithmic scales. Only one patient received all 4 consecutive doses of YF-H-2015005 as stipulated in the study protocol, and hence the PK parameters of 
Table 4. Summary of safety

\begin{tabular}{ll}
\hline & Number (\%) \\
\hline Adverse event & $15(100.0)$ \\
Treatment-related adverse event & $13(86.7)$ \\
Serious adverse event & $0(0.0)$ \\
Treatment-related serious adverse event & $0(0.0)$ \\
Common treatment-related adverse events & \\
Increased lactate dehydrogenase & $10(66.7)$ \\
Hot flashes & $2(13.3)$ \\
Diarrhea & $1(6.7)$ \\
Insomnia & $1(6.7)$ \\
Hyperhidrosis & $1(6.7)$ \\
\hline
\end{tabular}

\section{Discussion}

Autologous HSCT is the standard therapeutic modality for patients with NHL [9-12]. The successful mobilization of PB stem cells and adequate stem cell collection are of critical importance, as the infused $\mathrm{CD}^{+}{ }^{+}$cell dose can affect the engraftment of platelet and neutrophils [13]. The optimal amount of CD34 ${ }^{+}$ cells that causes rapid and sustained recovery is determined to be $>5 \times 10^{6}$ cells $/ \mathrm{kg}$, while the minimum threshold amount is deemed as $>2 \times 10^{6}$ cells $/ \mathrm{kg}$ [14]. However, failure to achieve sufficient HSC mobilization represents a great challenge in clinical practice, especially for supporting subsequent high-dose therapy and autologous HSCT [15]. In a retrospective study [16] conducted on 1,775 patients who underwent stem cell mobilization, $47 \%$ of the patients had a suboptimal stem cell collection (CD34 ${ }^{+}$ cell level $<5 \times 10^{6}$ cells $/ \mathrm{kg}$ ), among which, $25 \%$ had a low collection $\left(2-5 \times 10^{6}\right.$ cells $\left./ \mathrm{kg}\right), 10 \%$ had a poor collection $\left(<2 \times 10^{6}\right.$ cells $\left./ \mathrm{kg}\right)$, and $12 \%$ failed collection. Hence, there remains an urgent need to develop more effective, safer, and better tolerated treatment regimens for the mobilization of HSCs.

The greater effectiveness of using plerixafor in combination with G-CSF compared to G-CSF treatment alone has been demonstrated by a double-blind, randomized, placebo-controlled study [17]. A total of $298 \mathrm{NHL}$ patients were allocated to receive G-CSF in combination with plerixafor or a placebo. The proportion of NHL patients who achieved the primary endpoint $\left(\geq 5 \times 10^{6} \mathrm{CD}^{+} 4^{+}\right.$ cells $/ \mathrm{kg}$ ) was higher in plerixafor group $(59 \%)$ than in placebo group (20\%). It has been reported that plerixafor exhibits similar efficacy in a Chinese population [18]. In this randomized, double-blind study involving 101 patients with NHL, the number of patients who achieved a target collection of $>5 \times 10^{6}$ CD $34^{+}$cells $/ \mathrm{kg}(62 \%$ vs. $20 \%)$ or $>2 \times 10^{6}{\mathrm{CD} 34^{+}}^{+}$ cells $/ \mathrm{kg}$ ( $88 \%$ versus $66 \%$ ) and underwent autologous transplantation ( $88 \%$ versus $68 \%$ ) was higher in the plerixafor arm when compared to those in the placebo arm. Based on these prospective randomized studies, pre-emptive use of plerixafor for those patients at risk for HSC mobilization failure has been recommended in clinical practice guidelines $[14,15,19]$.

In the present study, the PK and PD of YF-H-2015005 in patients with NHL were evaluated. The PK findings revealed that YF-H-2015005 was rapidly absorbed and eliminated, with a mean $t_{1 / 2}$ of 5.04 hours. Moreover, the numbers of $\mathrm{CD}^{+} 4^{+}$cells in PB samples increased significantly after YF-H-2015005 administration. Treatment with YF-H-2015005 could result in a mean 2.9-fold elevation of PB CD34+ cells within 10 hours following YF-H-2015005 administration, by which the mean absolute PB CD34 ${ }^{+}$ cells elevated from 27.2 to 76.5 cells $/ \mu L$. Moreover, 14 (93\%) patients achieved the endpoint for $\mathrm{CD}^{+} 4^{+}$cell collection $\left(\geq 2 \times 10^{6}\right.$ cells $\left./ \mathrm{kg}\right)$, with a median $\mathrm{CD} 34^{+}$cell count of $3.35 \times 10^{6}$ cells $/ \mathrm{kg}$. These findings of PK and PD data supported the use of YF-H-2015005 dosing regimen and determined the optimal timing of apheresis.

More importantly, the target cell collection was obtained in $67 \%$ of patients after a single apheresis session and in $27 \%$ of patients after 2 apheresis sessions, which could lead to a reduction in health care costs (e.g., apheresis and hospitalization costs). Only 1 patient did not achieve the minimum target of $\geq 2 \times 10^{6} \mathrm{CD} 4^{+}$cells $/ \mathrm{kg}$ required for HSCT; however, similar to the other 14 patients, this patient exhibited a $\geq 2$-fold elevation in PB CD34+ ${ }^{+}$cells. In addition, all AEs were mild and transient with no SAEs reported. These findings indicated that YF-H-2015005 is an effective and safe agent for mobilizing HSCs in NHL patients.

In conclusion, our findings clearly demonstrated the potential of YF-H-2015005 as a safe, effective agent for HSC mobilization and subsequent collection by apheresis in patients with NHL. The rapid time course of YF-H-2015005-induced CD $34^{+}$cell mobilization as well as the relative paucity of adverse effects further supported the clinical applicability of this agent. The magnitude of an increased amount of $\mathrm{PB} \mathrm{CD} 34^{+}$cells after treatment with $0.24 \mathrm{mg} / \mathrm{kg}$ YF-H-2015005 was sufficient to indicate the clinical significance of this agent for mobilizing HSCs.

\section{Abbreviations}

AE: adverse event; $\mathrm{AUC}_{0-10 \mathrm{~h}}$ area under the curve from time 0 to $10 \mathrm{~h} ; \mathrm{AUC}_{0-24 \mathrm{~h}}$ : area under the curve from time 0 to $24 \mathrm{~h}$; $\mathrm{AUCO}_{-\infty}$ : area under the curve from time 0 to infinity; $\mathrm{AUC}_{\mathrm{Ss}}$ : steady-state area under the curve; $\mathrm{C}_{\mathrm{av}}$ : average steady-state plasma drug concentration; $C L$ : apparent clearance; $\mathrm{C}_{\max }$ : maximum observed concentration; $\mathrm{C}_{\text {ss.max }}$ : steadystate peak concentration; $\mathrm{C}_{\mathrm{ss}, \mathrm{min}}$ : steady-state trough concentration; CXCR4: CXC chemokine receptor 4; ECOG: Eastern Cooperative Oncology Group; Fl: 
coefficient of fluctuation; G-CSF: granulocyte colony stimulating factor; HSCs: hematopoietic stem cells; NHL: non-Hodgkin's lymphoma; PB: peripheral blood; PD: pharmacodynamics; PK: pharmacokinetic; SDF-1: stromal cell-derived factor-1; Ra: accumulation ratio; SAE: serious $\mathrm{AE}$; $\mathrm{t} 1 / 2$ : apparent terminal half-life; $\mathrm{T}_{\max }$ : observed maximum concentration; $\mathrm{V}_{\mathrm{z}}$ : apparent volume of distribution.

\section{Supplementary Material}

Supplementary tables.

http://www.jcancer.org/v11p5635s1.pdf

\section{Acknowledgements}

The authors wish to thank all participating patients, hemato-oncologists, pathologists, statisticians and the team of HSC mobilisation and apheresis for their invaluable contributions to this research project.

\section{Competing Interests}

Shufang Wang is an employee of Hefei Yifan Biopharmaceuticals Inc. The other authors declare that they have no conflicts of interest.

\section{References}

1. Karpova D, Ritchey JK, Holt MS, et al. Continuous blockade of CXCR4 results in dramatic mobilization and expansion of hematopoietic stem and progenitor cells. Blood. 2017;129:2939-2949.

2. Müller TA, Pennisi S, Zwick A, et al. PIM1 inhibition effectively enhances plerixafor-induced HSC mobilization by counteracting CXCR4 upregulation and blocking CXCL12 secretion. Leukemia. 2019;33:1296-1301.

3. Kawaguchi N, Zhang TT, Nakanishi T. Involvement of CXCR4 in Normal and Abnormal Development. Cells. 2019;8(2):185.

4. Sugiyama T, Omatsu Y, Nagasawa T. Niches for hematopoietic stem cells and immune cell progenitors. Int Immunol. 2019;31:5-11.

5. Dar A, Schajnovitz A, Lapid K, et al. Rapid mobilization of hematopoietic progenitors by AMD3100 and catecholamines is mediated by CXCR4-dependent SDF-1 release from bone marrow stromal cells. Leukemia. 2011;25:1286-1296.

6. Liles WC, Broxmeyer HE, Rodger E, et al. Mobilization of hematopoietic progenitor cells in healthy volunteers by AMD3100, a CXCR4 antagonist. Blood. 2003; 102:2728-2730

7. Stewart DA, Smith C, MacFarland R, Calandra G. Pharmacokinetics and pharmacodynamics of plerixafor in patients with non-Hodgkin lymphoma and multiple myeloma. Biol Blood Marrow Transplant. 2009;15:39-46.

8. Brave M, Farrell A, Ching Lin S, et al. FDA review summary: Mozobil in combination with granulocyte colony-stimulating factor to mobilize hematopoietic stem cells to peripheral blood for collection and subsequent autologous transplantation. Oncology. 2010;78:282-8.

9. Liu W, Ji X, Song Y, et al. Improving survival of 3760 patients with lymphoma: Experience of an academic center over two decades. Cancer Med. 2020;9(11):3765-3774

10. Philip T, Guglielmi C, Hagenbeek A, et al. Autologous bone marrow transplantation as compared with salvage chemotherapy in relapses of chemotherapy-sensitive non-Hodgkin's lymphoma. N Engl J Med. 1995;333:1540-5

11. Wu M, Wang X, Xie Y, et al. Outcome and Prospective Factor Analysis of High-dose Therapy Combined with Autologous Peripheral Blood Stem Cell Transplantation in Patients with Peripheral T-cell Lymphomas. Int J Med Sci. 2018;15(9):867-874

12. Liu W, Liu J, Song Y, et al. Burden of Lymphoma in China, 2006-2016: An Analysis of the Global Burden of Disease Study 2016. J Hematol Oncol. 2019;12(1):115

13. Hopman RK, DiPersio JF. Advances in stem cell mobilization. Blood Rev. 2014;28:31-40

14. Duong HK, Savani BN, Copelan E, et al. Peripheral blood progenitor cell mobilization for autologous and allogeneic hematopoietic cell transplantation: guidelines from the American Society for Blood and Marrow Transplantation. Biol Blood Marrow Transplant. 2014;20:1262-73.
15. Douglas KW, Gilleece M, Hayden $\mathrm{P}$, et al. UK consensus statement on the use of plerixafor to facilitate autologous peripheral blood stem cell collection to support high-dose chemoradiotherapy for patients with malignancy. J Clin Apher. 2018;33:46-59.

16. Gertz MA, Wolf RC, Micallef IN, Gastineau DA. Clinical impact and resource utilization after stem cell mobilization failure in patients with multiple myeloma and lymphoma. Bone Marrow Transplant. 2010;45:1396-403.

17. DiPersio JF, Micallef IN, Stiff PJ, et al. Phase III prospective randomized double-blind placebo-controlled trial of plerixafor plus granulocyte colony-stimulating factor compared with placebo plus granulocyte colony-stimulating factor for autologous stem-cell mobilization and transplantation for patients with non-Hodgkin's lymphoma. J Clin Oncol. 2009;27:4767-73.

18. Zhu J, Huang $\mathrm{H}$, Chen $\mathrm{H}$, et al. Plerixafor and granulocyte-colony-stimulating factor for mobilization of hematopoietic stem cells for autologous transplantation in Chinese patients with non-Hodgkin's lymphoma: a randomized Phase 3 study. Transfusion. 2018;58:81-87.

19. Mohty M, Hübel K, Kröger N, et al. Autologous haematopoietic stem cell mobilisation in multiple myeloma and lymphoma patients: a position statement from the European Group for Blood and Marrow Transplantation. Bone Marrow Transplant. 2014;49(7):865-872. 